\section{PTU-250 MANAGEMENT OF ANTICOAGULANT AND ANTIPLATELET THERAPY IN PATIENTS UNDERGOING ENDOSCOPIC PROCEDURES: COMPLIANCE WITH EXISTING GUIDELINES}

doi:10.1136/gutjnl-2012-302514c.250

0 Patani, ${ }^{*}$ S Khalid. Department of Gastroenterology, Warrington and Halton Hospitals NHS Trust, Warrington, UK

Introduction It is not uncommon for patients on anticoagulants and antiplatelets to undergo endoscopic procedures. The main issue in such cases is balancing the risk of gastro-intestinal bleed and the need for anticoagulation. Recognising this, the British Society of Gastroenterology (BSG) issued in 2008 practice guidelines on the management of anticoagulants and antiplatelet therapy in patients undergoing endoscopic procedures. Aspirin, Clopidogrel and Warfarin are the most commonly used antiplatelets and anticoagulants in the UK. Aspirin is recommended to be continued for all endoscopic procedures while Clopidogrel and Warfarin should be tailored to the bleeding risk category of the procedure and the thrombotic risk of the patient's condition. Our clinical practice was retrospectively audited to assess adherence to the BSG guidelines.

Methods All patients on antiplatelets and anticoagulants undergoing endoscopic procedures except ERCP were included in the audit. Data were collected from case notes, endoscopy records and the Éclair pathology reporting system.

Results 48 patients were identified to have 52 procedures with 37 (64\%) on Aspirin alone, 6 (10\%) on Clopidogrel alone, $9(16 \%)$ on Warfarin alone, 4 (7\%) on Aspirin and Clopidogrel and $2(3 \%)$ on Aspirin and Warfarin. 16/37 (43\%) in Aspirin alone group had it stopped inappropriately for 3-7 days. 3/6 (50\%) Clopidogrel alone patients had it stopped while having one high risk procedure and two low risk procedures. Warfarin was stopped inappropriately in 7/ $9(78 \%)$ patients undergoing eight procedures.

Conclusion Endoscopic procedures have evolved over the years to include increasingly complex and invasive procedures carrying risk of serious complications including bleeding. Also significant proportions of patients are on multiple medications including anticoagulants and antiplatelets. It is therefore essential that appropriate management plan for the anticoagulant and antiplatelet therapy should be made to minimise the haemorrhagic and thromboembolic risk when requesting endoscopic procedures. The findings of this audit underline significant non-compliance to existing guidelines with potential serious implications for the standard of patient care. While endoscopy is performed by trained endoscopists, the procedures are requested by clinicians with variable knowledge and awareness of the existing BSG guidelines. This hurdle can be overcome using a combination of raising awareness through educational events and modification of the endoscopy requesting software by including a link to the anticoagulation management guideline flow chart making it accessible to the clinicians at the time of requesting. We hope these measures will result in improvement of service and make endoscopy safer in our hospital.

Competing interests None declared.

\section{PTU-251 IMPACT OF APPLYING CURRENT BSG GUIDELINES ON COLORECTAL CANCER SCREENING AND SURVEILLANCE COLONOSCOPY PROCEDURES}

doi:10.1136/gutjnl-2012-302514c.251

R Sringeri, ${ }^{*}$ M Smith, S de Silva. Department of Gastroenterology, The Dudley Group NHS Foundation Trust, Birmingham, UK

Introduction The demand for colonoscopy is ever increasing, with greater pressures on endoscopy units. In order to provide a more efficient and timely service we conducted a review of our practice. Our aim was to audit colorectal cancer screening and surveillance colonoscopy procedures and determine the impact of the updated BSG guidelines (2010) on service provision. ${ }^{1}$

Methods The clinical letters, pathology reports and previous endoscopy reports were reviewed for all patients scheduled for surveillance colonoscopy over a period of 3 years (2010-2012). Data gathered included patient demographics, indication for procedure and outcome when audited against the current BSG guidelines. If the procedure did not meet the current guidelines with respect to indication or timing, the responsible clinician contacted both patient and GP, offering a clinic appointment to discuss further if needed.

Results A total of 354 colonoscopy requests were reviewed. Median age of the group was 62 years (range 22-98 years), male to female ratio 1.7:1. Indications included polyp surveillance 214 (60\%), IBD surveillance 55 (15\%), family history of colon cancer 50 (14\%), highrisk disease and family groups $17(5 \%)$, previous colon cancer $9(3 \%)$ and other indications 9 (3\%). 152 (43\%) were appropriate when audited against the current guidelines with no changes made to their procedures. Of the remaining 202, $106(52 \%)$ had the time to followup procedure extended (mean 3.25 years), 73 (37\%) did not meet the criteria for a repeat procedure and $23(11 \%)$ procedures were inappropriately delayed (mean $1.30 \mathrm{yrs}$ ). 125 colonoscopy requests were relevant to the updated guidelines for IBD surveillance, family history of colon cancer and high-risk disease and family groups. There were 57 IBD surveillance requests, of which 24 (42\%) had the time to follow-up procedure extended (mean $1.75 \mathrm{yrs}$ ). Out of 51 requests with family history of colorectal cancer, 23 (45\%) had the time to scheduled screening procedure extended (mean 8 yrs).

Conclusion Evaluating existing colonoscopy referrals against up to date BSG guidelines will contribute towards providing a more efficient colonoscopy service. In our practice a fifth of patients on the waiting list were inappropriate for further surveillance and a further third had the time to their procedure extended, therefore increasing capacity and efficiency to allow timely deliverance of procedures for those needing surveillance.

Competing interests None declared.

\section{REFERENCE}

1. Cairns S, et al. Gut 2010;59:666-90.

\section{PTU-252 CA19.9-ARE WE USING IT APPROPRIATELY AND IS IT COST EFFECTIVE?}

doi:10.1136/gutjnl-2012-302514c.252

${ }^{1} \mathrm{R}$ K Felwick, ${ }^{2} \mathrm{R}$ Olufadi, ${ }^{1} \mathrm{~S}$ Bridger. ${ }^{1}$ Department of Gastroenterology, Dorset County Hospital, Dorchester, UK; ${ }^{2}$ Department of Chemical Pathology, Dorset County Hospital, Dorchester, UK

Introduction CA19.9 is frequently used in the assessment of patients with suspected pancreatic cancer or cholangiocarcinoma. Although specific guidelines are not available, data suggests that it should not be used for screening and only used in conjunction with appropriate imaging. The aim of this study was to investigate the clinical utility and cost effectiveness of using CA19.9 as a marker for pancreatic cancer and cholangiocarcinoma in a district general hospital.

Methods A retrospective analysis was undertaken of all patients with a CA19.9 measurement over a period of 12 months. Data on liver biochemistry and abdominal imaging were collected. The results were compared to identify those patients in whom; (i) a positive CA19.9 result was associated with the presence of biliary or pancreatic malignancy (ii) those patients in whom CA19.9 was inappropriately requested. A cost analysis was undertaken. 